\title{
AVALIAÇÃO DE IMÓVEIS: A IMPORTÂNCIA DOS VIZINHOS NO CASO DE RECIFE*
}

\author{
Rubens Alves Dantas ${ }^{\S}$ \\ André Matos Magalhães ${ }^{\alpha}$ \\ José Raimundo de Oliveira Vergolino ${ }^{\dagger}$
}

\begin{abstract}
RESUMO
Tradicionalmente, na avaliação de imóveis, admite-se que as observações são independentes entre si. Contudo, dados associados à posição que ocupam no espaço são caracterizados pela dependência espacial. Seguindo a literatura internacional recente, este trabalho reconhece a importância da questão espacial e mostra como se pode diagnosticar e incorporar os efeitos espaciais na especificação de modelos hedônicos, ao mesmo tempo em que analisa o mercado imobiliário da cidade do Recife. Para realização dos testes empíricos e estimação dos modelos espaciais, são utilizados dados do Censo Demográfico do IBGE (2000) e de habitações financiadas pela Caixa Econômica Federal. Os resultados indicam que a consideração da questão espacial, com base em distâncias a pólos de influência, não é capaz de explicar completamente as variações dos preços em relação à localização da habitação, uma vez que existe uma verdadeira interação entre os dados, de forma que cada edifício funciona com um micropólo de influência sobre os seus vizinhos. Isso significa dizer que as negociações de compra dos apartamentos não ocorrem de forma independente, como considerado nos modelos tradicionalmente estimados, mas que existe uma verdadeira interação entre estes preços, de forma que uma negociação de um apartamento por um preço elevado tenderá a elevar os preços dos imóveis vizinhos. A solução para estimação segura da equação de preços hedônicos, na presença de efeitos espaciais, deve ser feita com base na econometria espacial, levando em consideração todas as interações espaciais possíveis entre os dados e servindo como uma proxy para variáveis locacionais não consideradas explicitamente no modelo.
\end{abstract}

Palavras-chave: avaliação de imóveis, modelo hedônico, econometria espacial.

\section{ABSTRACT}

Traditionally, in the assessment of real state values it is assumed that the observations are independent. However, data associated with the position that they occupy in the space are characterized by the spatial dependence. Following the recent international literature, this paper recognizes the importance of the space and shows how it can be incorporated in the specification of hedonic model at the same time it analyzes the real state market of the city of Recife. The data for the empirical test are derived from the IBGE Demographic Census (2000) and from the Caixa Econômica Federal database. The results indicate that considering space with base in distances to influence poles is not enough to completely explain the price variations in relation to the location, once there is a true interaction among the data, so that each building influences its neighbors' price. This means that, contrary to the traditional models hypothesis, the apartment transactions do not occur in an independent fashion. The solution for this problem is to estimate the model using the spatial econometric tools and taking into consideration all possible spatial interactions in the data.

Key words: housing price, hedonic price model, spatial econometrics.

JEL Classification: R20, C21.

* Os autores (Magalhães e Vergolino) agradecem o apoio do CNPq na elaboração desse trabalho.

$\S$ Doutor em Economia pelo PIMES-UFPE. Professor do Departamento de Engenharia Civil da Universidade Federal de Pernambuco e da Escola Politécnica de Pernambuco. E-mail: rubens@dantas.eng.br.

a Professor do Departamento de Economia da Universidade Federal de Pernambuco, Datamétrica, Recife, Brasil e Regional Economics Applications Laboratory (REAL) University of Illinois, Urbana, Illinois, USA. E-mail: magalhaes@ufpe.br.

$\dagger \quad \mathrm{PhD}$. em Economia pela Universidade de Illinois. Professor da Faculdade Boa Viagem e aposentado do Departamento de Economia da Universidade Federal de Pernambuco. E-mail: jose.vergolino@globo.com.

Endereço para contato: Rua Marechal Rondon, 300 - Casa Forte-Recife - Pe - CEP: 52061-050 - Brasil.

Recebido em março de 2006. Aceito para publicação em junho de 2007. 


\section{INTRODUÇão}

O valor de mercado do bem habitação é um parâmetro importante para tomada de decisão no setor público ou privado. Desta forma, é fundamental que se disponha de avaliações confiáveis. Por outro lado, a habitação é um bem composto por diversos serviços, chamados de serviços de habitação, correspondentes às suas características estruturais e locacionais, cujos preços individuais não são observados no mercado. O conhecimento do preço marginal que o consumidor está disposto a pagar por um determinado serviço de habitação, também chamado de preço implícito ou hedônico, pode ser de grande interesse para o empreendedor, na medida em que pode ajudar na análise de custo-benefício, como também para elaboração e implantação de políticas habitacionais e urbanas, uma vez que, no longo prazo, são as preferências do consumidor que determinam a configuração das cidades.

A avaliação de imóveis, e dos seus preços implícitos ou hedônicos, tem sido realizada, no Brasil, utilizando-se a técnica da regressão hedônica ${ }^{1}$ por meio da Econometria Tradicional. ${ }^{2}$ Por este processo faz-se uma regressão dos preços dos imóveis sobre as suas características estruturais (área privativa, número de cômodos, vagas na garagem, idade, conservação, padrão construtivo, etc.), locacionais (cidade, região, bairro, distâncias a pólos de influência, etc.) e econômicas (forma de pagamento, época da transação, natureza do evento, etc.), admitindo-se as observações independentes entre si. Contudo, dados associados à posição que ocupa no espaço, como é o caso dos dados imobiliários, são caracterizados pela dependência ou heterogeneidade espacial (Anselin, 1988). Neste caso, os resultados obtidos pela metodologia tradicional não são capazes de explicar com fidelidade o comportamento do mercado imobiliário, uma vez que podem apresentar problemas de tendenciosidade, inconsistência ou ineficiência. A solução é proceder à análise com base nos Modelos Espaciais, isto é, modelos estimados pela metodologia denominada Econometria Espacial. ${ }^{3} \mathrm{~A}$ Econometria Espacial foi desenvolvida na década de 70 e recebeu grande impulso nas ampliações realizadas por Anselin (1988), principalmente na parte aplicada, com o desenvolvimento da ferramenta computacional denominada SpaceStat (Anselin, 1990).

A literatura internacional reconhece a importância da questão espacial na modelagem de preços hedônicos, como também na avaliação do bem habitação, e tem tratado o problema de diversas formas. Basu e Thibodeau (1998), por exemplo, utilizam covariogramas e semicovariogramas para testar os efeitos espaciais; Can (1990) utiliza um índice de vizinhança construído a partir de análises fatoriais. Outros exemplos podem ser encontrados em Can (1992), Dubin (1992), Olmo (1998), Garcia et al. (2002) e Olmo e Guervós (2002).

O trabalho elaborado por Dantas et al. (2001) para o Brasil representa a primeira tentativa de incorporar a questão espacial à avaliação de imóveis. Mais especificamente, tal estudo estima um modelo espacial para uma região da cidade do Recife, com uma amostra de apartamentos situados em 59 edifícios residenciais, distribuídos em quatro bairros e encontra indicações de autocorrelação espacial. Em expansões deste trabalho, Dantas et al. (2002a), Dantas et al. (2002b) e Magalhães e Dantas (2002), com ampliação da amostra e do número de bairros, foram encontrados resultados

1 A regressão hedônica foi popularizada por Griliches no início dos anos 1960. Todavia, Court (1939) foi pioneiro nessa questão - Goodman (1998) discute a importância do trabalho de Court. Rosen (1974), por sua vez, apresenta um excelente trabalho no qual o modelo hedônico é exposto em detalhes e serve como base para o que será apresentado a seguir.

2 Usa geralmente como ferramenta estatística o modelo clássico de regressão.

3 Neste caso, a ferramenta estatística utilizada é a regressão espacial. 
mais consistentes e que reforçam a presença de efeitos espaciais em dados habitacionais na cidade do Recife.

Isto posto, pode-se afirmar que há grande probabilidade de os resultados dos estudos realizados até o momento, com base na econometria tradicional, serem tendenciosos, ineficientes ou inconsistentes, tendo em vista que negligenciaram a presença de efeitos espaciais nos dados. Assim, acredita-se que a utilização da econometria espacial pode ajudar na especificação e estimação dos modelos de preços hedônicos e, conseqüentemente, nos resultados das avaliações dos imóveis.

Este trabalho pretende atingir dois objetivos: um relacionado aos aspectos metodológicos e outro de natureza empírica. O de caráter metodológico consiste em mostrar como se pode diagnosticar e incorporar os efeitos espaciais na especificação de modelos de preços hedônicos, utilizandose a Econometria Espacial. O segundo trata da pesquisa aplicada ao estudo da economia urbana, mais concretamente a análise microeconômica do mercado imobiliário da cidade do Recife, capital do Estado de Pernambuco, uma das mais importantes cidades do País. ${ }^{4}$ Para realização dos testes empíricos e estimação dos modelos espaciais, serão utilizados dados do Censo Demográfico do IBGE (2000) e de habitações financiadas pela Caixa Econômica Federal.

O trabalho será composto por cinco seções, além desta Introdução. Na Seção 2, apresenta-se a base teórica da estimação empírica que será realizada nas seções seguintes. Na Seção 3, faz-se um diagnóstico da dependência espacial entre a renda dos bairros do Recife, utilizando dados do Censo Demográfico do IBGE (2000). A Seção 4 ocupa-se da estimação de um modelo espacial de preços hedônicos, utilizando-se o banco de dados de apartamentos financiados pela CAIXA. Com base neste modelo, analisa-se o comportamento do consumidor perante as características da cesta de habitação com que se defronta no mercado imobiliário. Finalmente, na Seção 5, são apresentadas as conclusões e recomendações.

\section{REGRESSÃO HEDÔNICA}

Por ser o imóvel um bem heterogêneo, a sua avaliação é feita usualmente com modelos de preços hedônicos. Nestes modelos, busca-se extrair a importância marginal de cada atributo do imóvel na determinação do seu preço. Os preços hedônicos são definidos como os preços implícitos dos atributos e são revelados para os agentes econômicos por meio dos preços observados dos produtos diferenciados e as quantidades específicas das características associadas a eles (Rosen, 1974). No caso de imóveis, são considerados como atributos, em geral, características decorrentes dos seus aspectos físicos (área, padrão construtivo, número de vagas na garagem, etc) e de localização (bairro onde se situa o imóvel, distância a pólos de influência, etc.), bem como de aspectos econômicos (condições de pagamento do imóvel, natureza do evento: em oferta ou efetivamente vendido, etc.).

$\mathrm{Na}$ formulação básica do modelo, são considerados os consumidores, que adquirem uma unidade de um bem composto, e o setor de produção. Os consumidores resolvem o seguinte problema:

$$
\operatorname{Max} U\left(x, z_{i}\right), \text { s.a. } y=x+P\left(z_{i}\right), \quad i=1,2, \ldots, n
$$

onde $U(\cdot)$ é a função de utilidade, por suposição estritamente côncava, $x$, tomado como nume-

4 Recife é a $7^{\mathrm{a}}$ capital do País em população e a $8^{\mathrm{a}}$ em número de domicílios. 
rário, representa todos os bens consumidos com exceção do bem composto, representado por $z_{i}$, $y$ é a renda e $P(z)$ é a função de preços hedônicos. Do problema de maximização, tem-se que as condições de primeira ordem implicam

$$
\frac{\partial P}{\partial Z_{i}} \equiv P_{i}=\frac{U_{Z i}}{U_{X}}
$$

onde $P_{i}$ é o preço hedônico do atributo $i^{5}$

Seguindo Rosen, $\theta\left(Z_{i} ; u, Y\right)$ é definida como uma função dispêndio, tal que

$$
U\left(y-\theta, z_{i}\right)=u
$$

A equação (3) indica que o gasto que o consumidor está disposto a realizar, para valores alternativos de $z_{i}$ a um dado nível de utilidade $\mu$ e renda $y$ é $\theta\left(z_{i} ; u, y\right)$. Derivando $\theta$ com relação à $z$ em (3) tem-se que:

$$
\theta_{Z_{i}}=\frac{U_{Z_{i}}}{U_{X}}
$$

onde $\theta_{Z i}$ indica o preço de reserva para uma unidade adicional de $z_{i}{ }^{6}$

Nesta estrutura, por um lado, $\theta\left(z_{i} ; u, y\right)$ representa o valor que o consumidor está disposto a pagar por $z$, dados utilidade e renda, enquanto que $P(z)$ é o preço mínimo que ele deve pagar no mercado. Conseqüentemente, a utilidade é maximizada quando

$$
\theta\left(z_{i}^{\prime} ; u^{\prime}, y\right)=P\left(z_{i}^{\prime}\right) \quad \text { e } \quad \theta_{Z_{i}}\left(z_{i}^{\prime}, u^{\prime}, y\right)=P_{i}\left(z_{i}^{\prime}\right), \quad i=1,2, \ldots, n
$$

onde $z^{\prime}$ e $u^{\prime}$ são as quantidades ótimas.

Por outro lado, o problema do produtor é o de determinar o número de unidades, $M(z)$, que ele produzirá de forma a maximizar os seus lucros. A função de custo total é dada por $C(M, z ; \beta)$, onde $\beta$ é um parâmetro de deslocamento que reflete fatores como os parâmetros da função de produção. ${ }^{8}$ Cada firma resolve o seguinte problema:

$$
\underset{M, z}{\operatorname{Max}} \pi=M P(z)-C\left(M, z_{i}\right)
$$

As condições de primeira ordem do problema implicam que a receita marginal de cada atributo deve ser igual a seu custo marginal e que a produção irá até o ponto que a receita unitária é igual ao custo marginal de produção, ou seja: ${ }^{9}$

$$
P_{i}=\frac{C_{z i}\left(M, z_{i}\right)}{M}
$$

5 As condições de segunda ordem são satisfeitas sob hipóteses simples (ver Rosen, 1974).

$6 \theta_{z i}$ pode ser interpretado como a taxa marginal de substituição entre $Z_{i}$ e dinheiro ou ainda como o valor marginal implícito para o consumidor de $Z_{i}$, dados o nível de utilidade e a renda.

7 Uma apresentação gráfica e extensão do modelo são apresentadas em Rosen (1974).

8 C é uma função convexa com $C(0, z)=0$ e $C M>0, C_{z i}>0$. As firmas são competitivas e tomam os preços como dados.

9 As condições de segunda ordem são garantidas por algumas restrições adicionais (ver Intriligator, 1971). 


$$
P(z)=C_{M}\left(M, z_{i}\right)
$$

Como no caso da função dispêndio dos consumidores, uma função oferta, $\phi\left(z_{i} ; \pi, \beta\right)$, é definida para os produtores, indicando os preços unitários que as firmas estão dispostas a aceitar nas várias composições do produto a um lucro constante, quando a produção é otimamente escolhida. Assim sendo, $\phi\left(z_{i} ; \pi, \beta\right)$ é encontrada eliminando $M$ de

$$
\pi=M \phi-C\left(M, z_{i}\right)
$$

e

$$
\phi=C_{M}\left(M, z_{i}\right)
$$

e resolvendo para $\phi$ em termos de $z, \pi$ e $\beta$. Derivando (9) e (10) tem-se que: $\phi_{z_{i}}=C_{z_{i}} / M>0$ e $\phi_{\pi}=1 / M>0, \phi_{z_{i}}$ é o preço marginal de reserva de oferta para o atributo $i$ a um lucro constante. Dado que $\phi$ é o preço que a firma está disposta a aceitar para a composição $z$ ao lucro $\pi$, enquanto $P(z)$ é o preço máximo alcançável no mercado, o lucro máximo será alcançado por uma maximização equivalente do preço de oferta sujeito a restrição $P(z)=\phi$. Assim sendo, o lucro máximo e a composição ótima satisfazem as condições:

$$
P_{i}\left(z^{\prime}\right)=\phi_{z_{i}}\left(z_{i}^{\prime} ; \pi, \beta\right), \quad i=1,2, \ldots, n
$$

e

$$
P\left(z^{\prime}\right)=\phi\left(z_{i}^{\prime} ; \pi, \beta\right), \quad i=1,2, \ldots, n
$$

No equilíbrio do mercado existirá uma função $P(z)$, tal que as quantidades ofertadas e demandadas se igualam. Desta forma, no equilíbrio, $P(z)$ representa não só o preço de reserva do consumidor como também o preço de reserva da firma. ${ }^{10}$

Como dito acima, na estimação de preços hedônicos, busca-se extrair a importância marginal de cada atributo, z, do imóvel na determinação do seu preço. No caso de imóveis, são considerados como atributos as características decorrentes dos seus aspectos físicos e de localização, bem como de aspectos econômicos. A forma geral do modelo hedônico para o preço do imóvel pode ser dada por:

$$
P=f(L, S, N, A, t)
$$

onde $P$ é um valor de mercado estimado da propriedade, $L$ inclui as características do terreno, $S$ as características físicas do imóvel, $N$ representa as características de vizinhança, $A$ inclui variáveis de acesso e amenidades do imóvel e $t$ denomina o tempo em que as informações foram coletadas.

10 As condições para a existência do equilíbrio de mercado são apresentadas e discutidas em Rosen (1974). 


\subsection{Equação empírica}

Seguindo Basu e Thibodeau (1998), a especificação para a forma funcional da eq. (13) aqui utilizada será a exponencial, dada por:

$$
P=e^{X \beta+\varepsilon}
$$

onde $P$ é o valor do imóvel, $X$ é a matriz de características do imóvel, $\beta$ é um vetor de coeficientes e $\varepsilon$ representa os resíduos. Pode-se, então, utilizar o método de mínimos quadrados ordinários para estimar a equação transformada na forma semilog: ${ }^{11}$

$$
\ln P=X \beta+\varepsilon
$$

onde $\varepsilon \sim N\left(0, \sigma^{2} I\right)$ de forma que $\ln P \sim N\left(X \beta, \sigma^{2} I\right)$. Uma relação interessante com respeito ao modelo apresentado na eq. (15) é que os coeficientes das variáveis multiplicados por 100 podem ser interpretados como o efeito porcentual de cada variável independente sobre a variável dependente. $^{12}$

Uma hipótese comumente imposta na análise dos preços é a de que as observações são independentes. Entretanto, como na avaliação de imóveis os dados são distribuídos no espaço, surge a possibilidade de que existam erros de medidas em relação à localização do imóvel como também de efeitos de interação, difusão e "spillovers" espaciais. A literatura internacional tem reconhecido a importância do espaço na determinação dos preços dos imóveis e tem tratado o problema de diversas formas. Basu e Thibodeau (1998), por exemplo, utilizam covariogramas e semicovariogramas espaciais para corrigir as estimativas dos mínimos quadrados ordinários. Can (1990) utiliza um índice de vizinhança construído a partir de análise fatorial. Outros exemplos podem ser encontrados em Can (1992), Dubin (1992) e Olmo (1998). O trabalho de Dantas et al. (2001) é uma das primeiras tentativas de incorporar a questão espacial à avaliação de imóveis no Brasil.

No caso da existência de efeitos espaciais, a metodologia tradicionalmente adotada torna-se inadequada, podendo levar o avaliador à obtenção de resultados não confiáveis. Uma das formas de se tratar da questão espacial é pela consideração explícita da autocorrelação espacial, ou dependência espacial, que pode ser incorporada no modelo de regressão representado na eq. (15), por meio da metodologia desenvolvida pela econometria espacial. A não consideração da dependência espacial, caso exista, pode gerar sérios problemas ao trabalho avaliatório, uma vez que os parâmetros estimados podem se apresentar tendenciosos, ineficientes e inconsistentes. Pode-se ainda enfatizar que esta metodologia é de grande importância para a engenharia de avaliações urbanas, principalmente quando se trata de trabalhos que envolvem avaliações em massa, como é o caso da elaboração de plantas genéricas de valores municipais, apresentando, assim, além da relevância teórica, uma grande importância prática.

11 A forma funcional semilog foi utilizada com o objetivo de estabilizar a variância e normalizar os resíduos. Este modelo tem sido largamente adotado para explicar o comportamento do mercado habitacional - ver Ermisch et al. (1996), Tiware et al. (1999), Olmo e Guervós (2002), Can (1992), Basu e Thibodeau (1998), Lucena (1985). Dantas e Cordeiro (1986, 1988, 2000 e 2001), utilizando as transformações de Box-Cox (1964), verificaram que esta forma funcional é a que melhor se ajusta ao mercado imobiliário do Recife.

12 Essa interpretação é válida para variáveis contínuas. No caso de variáveis dummy a interpretação é um pouco diferente, sendo o efeito porcentual dado por 100. $\left(e^{\beta}-1\right)$, como demonstrado por Halvorsen e Palmquist (1980). 
A autocorrelação espacial pode ser definida como sendo "a coincidência de valores semelhantes em locais semelhantes". Em um sentido mais amplo, o qual será adotado neste trabalho, autocorrelação espacial implica a ausência de independência entre observações em dados de corte transversal. Em outras palavras, ela pode ser interpretada como "a existência de uma relação funcional entre o que acontece em um certo ponto no espaço e o que acontece em outro lugar" (Anselin, 1988, p. 11). O problema causado pela presença de autocorrelação espacial é, basicamente, sua implicação de que a amostra contém menos informação que as partes que são não correlacionadas (Anselin e Bera, 1998).

A relação pode se originar como um problema de erro de medida, que surge do fato de que os dados para as variáveis de interesse são divididos em unidades artificiais, como Estados, municípios, cidades ou bairros, os quais freqüentemente não coincidem com a real dimensão espacial do fenômeno em consideração. Neste caso, é provável que efeitos de espalhamento ocorram e os erros em unidades diferentes serão, provavelmente, relacionados uns com os outros. A implicação desse tipo de dependência sobre os coeficientes estimados é que as estimativas dos mesmos não seriam eficientes. $^{14}$

Por outro lado, autocorrelação espacial pode se originar como resultado de uma verdadeira interação espacial entre as variáveis. Neste caso, o problema se torna mais interessante no contexto econométrico, sentido que os coeficientes estimados por meio do método de mínimos quadrados ordinários seriam viesados. No presente contexto, ou seja, de determinação da importância dos atributos no preço dos imóveis, tal fato poderia implicar uma superestimação ou subestimação de algumas características. ${ }^{15}$

Identificada a possibilidade da existência de dependência espacial entre as unidades em estudo, se faz importante incluir a dimensão espacial ao problema a ser tratado.

\subsection{Modelo de dependência no erro}

Como visto acima, uma das possíveis causas da autocorrelação espacial seria o problema relacionado aos erros de medida, ocasionados pelas divisões artificiais das unidades geográficas, no presente caso, os bairros que não necessariamente coincidem com a verdadeira dimensão do fenômeno observado. Este tipo de dependência espacial poderia também ser o resultado de alguma variável omissa que capturasse a dimensão espacial do problema, e tal ausência conduziria a erros espacialmente correlacionados.

Nesse sentido, a primeira modificação com relação à equação (15) seria considerar o termo de erro que segue um processo espacial autorregressivo, da seguinte forma:

$$
\varepsilon_{t}=\lambda \mathbf{W} \varepsilon_{t}+u_{t}
$$

onde $\lambda$ é um escalar que representa o coeficiente da correlação espacial do erro, W é uma matriz

13 Ver Anselin e Bera (1998). A noção de autocorrelação espacial foi introduzida por Cliff e Ord (1973).

14 Ver Anselin para uma demonstração formal do problema.

15 Além dos problemas mencionados acima, há também aqueles que se originam da falta de homogeneidade das próprias unidades espaciais. Unidades distintas (Estados, cidades, etc.) têm, por exemplo, tamanhos, formas, densidades diferentes e estas diferenças podem gerar erros de medida que podem causar heteroscedasticidade (ver Anselin e Bera, 1998). 
de pesos espaciais ${ }^{16}$ e $u_{t}$ é normalmente distribuído com média zero e desvio padrão constante. Substituindo (16) em (15) resulta na regressão do erro espacial

$$
\ln P=X \beta+(I-\lambda \mathbf{W})^{-1} u
$$

Nos casos em que a dependência espacial é do tipo da expressa em (16), a estimação da equação (15) por mínimos quadrados ordinários conduziria a estimativas não viesadas, mas ineficientes dos parâmetros, devido à estrutura não diagonal da matriz de variância dos resíduos (ver Anselin, 1988). Para obter estimativas eficientes dos parâmetros das equações, faz-se necessário utilizar o estimador baseado na função de verossimilhança

$$
L=\frac{n}{2} \ln (\pi)-\frac{n}{2} \ln \left(\sigma^{2}\right)+\ln |\mathbf{I}-\lambda \mathbf{W}|-\frac{1}{2 \sigma^{2}} \varepsilon^{\prime}(\mathbf{I}-\lambda \mathbf{W})^{\prime}(\mathbf{I}-\lambda \mathbf{W}) \varepsilon
$$

\section{$2.3 \mathrm{O}$ modelo de defasagem espacial}

A segunda possibilidade considerada é que a dependência espacial seja criada por meio de uma real interação espacial entre os preços dos imóveis, ou seja, existe um efeito de vizinhança na determinação dos preços. A eq. (15) passa a ser

$$
\ln P=X \beta+\rho \mathbf{W} \ln P+\varepsilon
$$

O coeficiente $\rho$ é um escalar que capta o efeito do preço dos vizinhos sobre o preço de cada imóvel e $\varepsilon$ segue uma distribuição normal $(0,1)$.

Esses modelos deverão ser estimados no que se segue, caso venha a se confirmar a existência de dependência espacial nos dados utilizados. Mais uma vez a estimação por mínimo quadrados ordinários não é adequada, sendo os parâmetros não viesados apenas se $\rho=0$. Para resolver esse problema, as equações acima devem ser estimadas por um estimador baseado na função de verossimilhança dada por ${ }^{17}$

$$
L=\frac{n}{2} \ln (\pi)-\frac{n}{2} \ln \left(\sigma^{2}\right)+\ln |\mathbf{I}-\rho \mathbf{W}|-\frac{1}{2 \sigma^{2}} \varepsilon^{\prime} \varepsilon
$$

No restante desse trabalho, os dados são apresentados e os resultados empíricos são analisados.

16 Esta matriz, normalmente conhecida como W, pode ser usada para capturar padrões de adjacência das unidades geográficas. No caso mais simples, uma matriz simétrica é definida como tendo o elemento $(i, j)$ igual a 1 se $i$ e $j$ são vizinhos e 0 no caso contrário. Por convenção, os elementos diagonais são iguais a zero, $w_{i i}=0$. A matriz de peso espacial pode ser padronizada pela linha, denominada pelo sobrescrito s, com cada um dos seus elementos que têm valor diferente de zero sendo definido por $w_{i j}^{s}=w_{i j} / \sum_{j} w_{i j}$. Nesta matriz, os elementos das linhas somam 1. Além de facilitar a interpretação dos pesos (que variam entre 0 e 1) como uma média dos valores dos vizinhos, esta manipulação assegura a comparabilidade entre modelos, dos parâmetros espaciais em muitos processos espaciais estocásticos (Anselin e Bera, 1998). Há ainda outras especificações mais complexas de matrizes de peso baseadas, por exemplo, em variáveis econômicas (ver Cliff e Ord, 1973 e 1981 e Case et al., 1993).

17 Uma derivação do estimador de verossimilhança pode ser encontrada em Anselin (1988). 


\section{UMA ANÁLISE DA DISTRIBUIÇÃO ESPACIAL DA RENDA NA CIDADE DO RECIFE}

Tendo em vista que a renda é um fator determinante na escolha da habitação, antes de se partir para a estimação da regressão hedônica, faz-se uma análise espacial da distribuição de renda dos chefes de família da cidade do Recife. Esta análise pode auxiliar na estimação do modelo de preços hedônicos, na definição de possíveis pólos de atração ou repulsão inseridos no contexto urbano, bem como ajudar na interpretação e leitura dos resultados. Dessa forma, nesta seção será realizada uma análise da dependência espacial da renda entre os bairros da cidade do Recife.

Existem duas formas de se diagnosticar a presença de efeitos espaciais em uma amostra: pela análise gráfica do variograma ou utilizando-se testes estatísticos específicos como os testes de Moran I e os testes LM (Multiplicador de Lagrange). ${ }^{18}$ No primeiro caso, a inferência espacial é realizada pelo processo denominado KRI ou de Krigagem, desenvolvido por Matheron (1965); no segundo caso, a modelagem espacial é realizada segundo a metodologia desenvolvida por Anselin (1988), apresentada na Seção 2. Neste trabalho, será utilizada, predominantemente, a metodologia de Anselin (1988). Uma boa resenha sobre a outra metodologia pode ser encontrada em Guervós (1999).

Para a realização do estudo, foram utilizadas informações fornecidas pelo censo demográfico do IBGE (2000) e do departamento de cartografia da UFPE, que forneceu as coordenadas geográficas dos centróides dos bairros $(\mathrm{X}, \mathrm{Y})$, em UTM. ${ }^{19}$ Para detectar a presença de efeitos espaciais, utiliza-se, inicialmente, a análise gráfica do variograma e, em seguida, testes de autocorrelação espacial, pela qual o diagnóstico é realizado pelos testes $\mathrm{LM}^{20}$ Robusto (erro) e LM Robusto (defasagem), sobre os resíduos de mínimos quadrados de um modelo ajustado a um polinômio de tendência do segundo grau, segundo metodologia desenvolvida por Anselin (1988). O modelo consiste em se fazer uma regressão da variável analisada sobre as coordenadas geográficas correspondentes ao centróide do bairro (X e Y), seus quadrados (X2 e Y2) e sua interação (XY). Este polinômio indicará as variações da variável analisada em grande escala espacial. Para evitar problemas graves de multicolinearidade, geralmente presentes neste tipo de modelo, utilizam-se as coordenadas transformadas em termos de desvios em relação à média (Olmo e Guervós, 2002).

Para a realização dos testes LM Robusto (erro) e LM Robusto foi necessário, inicialmente, construir uma matriz de vizinhança W, com 94 linhas e 94 colunas, onde cada elemento da matriz, $w_{i j}$, representa o inverso da distância entre os centróides dos bairros. Isto é, neste caso, a influência de cada bairro sobre o seu vizinho é medida pelo inverso da distância entre os seus centróides (X, Y). Assim, quanto maior esta distância, menor será a dependência espacial entre eles. Em seguida, a matriz é padronizada por linha, isto é, cada elemento da nova matriz padronizada é encontrado

18 Os principais testes utilizados para detectar a autocorrelação espacial são: Moran I (erro); LM Robusto (erro) e LM Robusto (defasagem). O teste de Moran I, apesar de ser um teste robusto, indica apenas a presença ou não de autocorrelação espacial, mas não identifica se o tipo de efeito é de erro ou de defasagem espacial, fator importante para a escolha do modelo. Por isso, neste trabalho, serão utilizados também outros testes, que são mais específicos: o LM (erro) Robusto para detectar efeitos de autocorrelação espacial no termo de erro; e o LM (defasagem) Robusto para verificar a presença de efeitos de defasagem espacial na variável dependente. Maiores detalhes podem ser encontrados Anselin(1988b). O teste LM (erro) Robusto é um teste assintótico realizado a partir da estatística $L M_{\lambda}=\left[\left(\varepsilon^{\prime} W \varepsilon\right) / \sigma^{2}\right]^{2} / \operatorname{tr}\left[\left(W^{\prime}+W\right) W\right]$, onde $\varepsilon$ é o vetor de resíduos de mínimos quadrados; W a matriz de pesos espaciais; $\sigma^{2}$ é estimativa de máxima verossimilhança da variância do modelo de mínimos quadrados; $n$ o número de dados da amostra e $t r$ o operador denominado traço da matriz, que tem distribuição Qui-quadrado com um grau de liberdade, sob a hipótese nula de não existência de autocorrelação espacial no termo erro. O teste LM (defasagem) Robusto é também um teste assintótico realizado a partir da estatística $L M_{\rho}=\tilde{d}_{\rho}^{2} / \tilde{T}_{1}=\left[\left(\varepsilon^{\prime} W y\right) / \tilde{\sigma}^{2}\right]^{2} / \tilde{T}_{1}$, onde $T_{1}=\left[(W X \beta)^{\prime} M(W X \beta)+T \sigma^{2}\right] / \sigma^{2}$, $T=\operatorname{tr}\left[\left(W^{\prime}+W\right) W\right]$ e $M=I-X\left(X^{\prime} X\right) X$. Esse teste tem distribuição Qui-quadrado com um grau de liberdade, sob a hipótese nula de não existência de defasagem espacial na variável dependente.

19 Sigla de Universal Transversa de Mercator.

20 Sigla de Lagrange Multiplicador. 
dividindo-se o seu valor pela soma dos valores dos elementos da linha a que pertence. Neste caso, os elementos da matriz padronizada podem ser interpretados como ponderadores de influência.

Para verificação da presença de efeitos de dependência espacial entre renda dos bairros do Recife, foi construído, inicialmente, um variograma experimental e ajustado ao mesmo um variograma teórico, do tipo gaussiano, que está plotado na Figura 1. Por este gráfico, pode-se observar que o alcance do variograma é de $3 \mathrm{~km}$. Ou seja, a variância espacial cresce até uma distância de cerca de $3 \mathrm{~km}$ entre os dados, havendo, a partir de então, uma tendência de estabilização da mesma. Este comportamento indica que o raio de influência de contágio espacial em relação à renda é de cerca de $3 \mathrm{~km}$, tornando-se desprezíveis estes efeitos, a partir deste limite. Assim, há evidências de que famílias com faixas de renda semelhantes tendem a morar próximas umas das outras, ou melhor, existe um agrupamento de bairros de rendas elevadas e também os bairros de padrão de rendas baixas têm vizinhos na mesma faixa de renda.

Figura 1 - Variograma experimental da renda média dos chefes de família da cidade do Recife, 2000

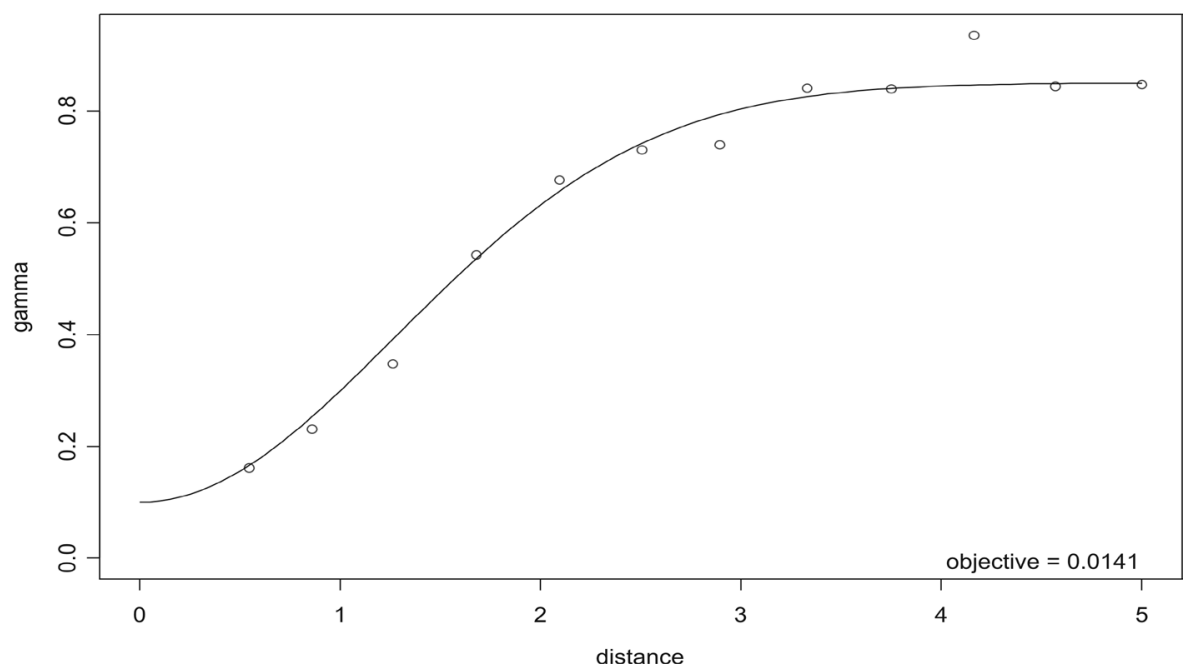

Fonte: IBGE - Censo Demográfico 2000.

Com base na estrutura definida pelo variograma, realiza-se a inferência espacial, por meio do processo de Krigagem, obtendo-se as curvas de isorenda mostradas na Figura 2, como também o mapa de distribuição de renda apresentado na Figura 3. 
Figura 2 - Mapa de isorenda da cidade do Recife, ano 2000

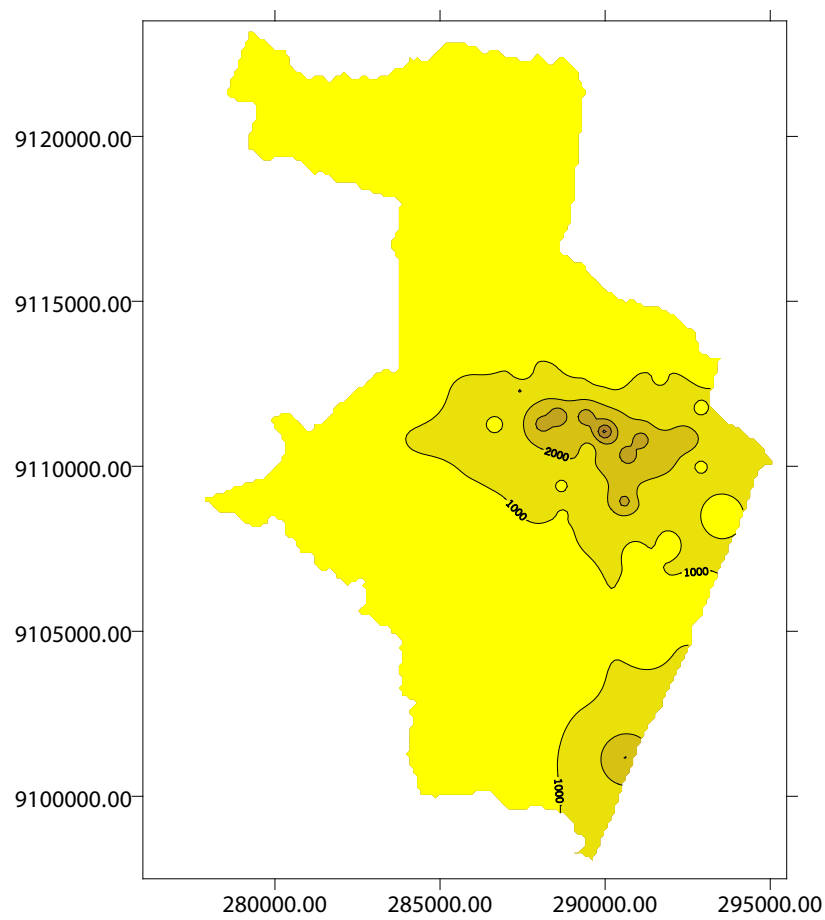

Fonte: IBGE - Censo Demográfico 2000.

Nota: no eixo horizontal, as coordenadas geográficas crescem no sentido oeste-leste e, no eixo vertical, estas coordenadas crescem no sentido sul-norte. A parte mais escura da figura representa a região de Boa Viagem na zona sul e a região da Jaqueira na zona centro-oeste.

\section{Figura 3 - Mapa de distribuição de renda da cidade do Recife, ano 2000}
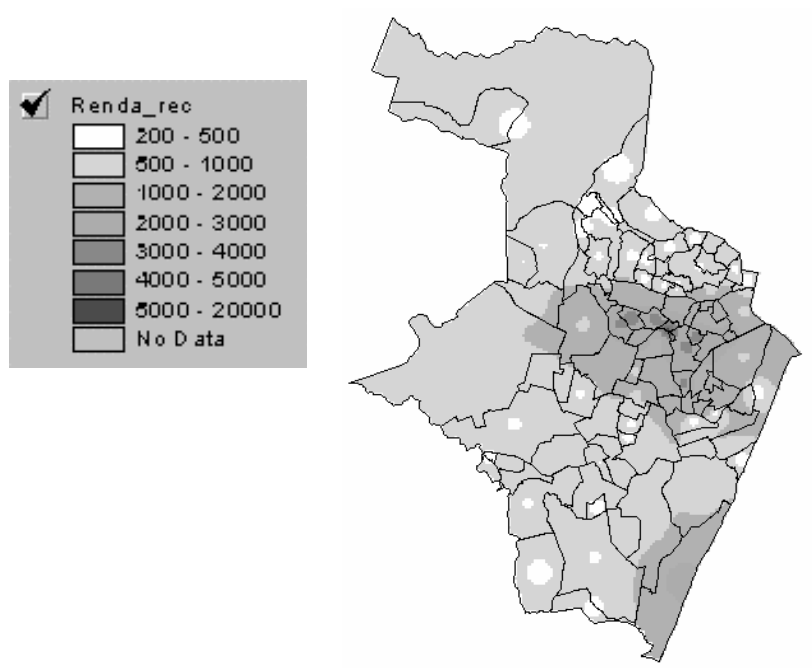

Fonte: IBGE - Censo Demográfico 2000.

Nota: no eixo horizontal, as coordenadas geográficas crescem no sentido oeste-leste e no eixo vertical, estas coordenadas crescem no sentido sul norte. A parte mais escura, na zona sul, representa a região de Boa Viagem e, na zona centro-oeste, a região da Jaqueira. 
Pelas Figuras 2 e 3 identifica-se claramente uma concentração de renda, representada pelas partes mais escuras das figuras, em torno de dois pólos de influência da cidade. Na zona sul, a praia de Boa Viagem e, conseqüentemente, o bairro de Boa Viagem, e, na zona norte, o parque da Jaqueira, com influência sobre os bairros da Jaqueira, Tamarineira, Graças, Parnamirim, Aflitos, Casa Forte e Espinheiro. Uma visão ainda mais clara deste efeito pode ser observada no mapa de curvas de nível de renda, mostrado na Figura 4.

A Figura 4 mostra a estrutura multicêntrica da cidade do Recife, deixando claro que o centro histórico da cidade não representa o pólo de maior influência da cidade, em termos de concentração da população de maior poder aquisitivo. Isto era um fato esperado e ocorre na maioria das cidades de grande porte do Brasil, em função dos imóveis localizados no centro da cidade serem bastante antigos, além das questões relacionadas com trânsito, poluição e falta de amenidades urbanas positivas.

Figura 4 - Mapa de curvas de nível de isorenda da cidade do Recife, ano 2000

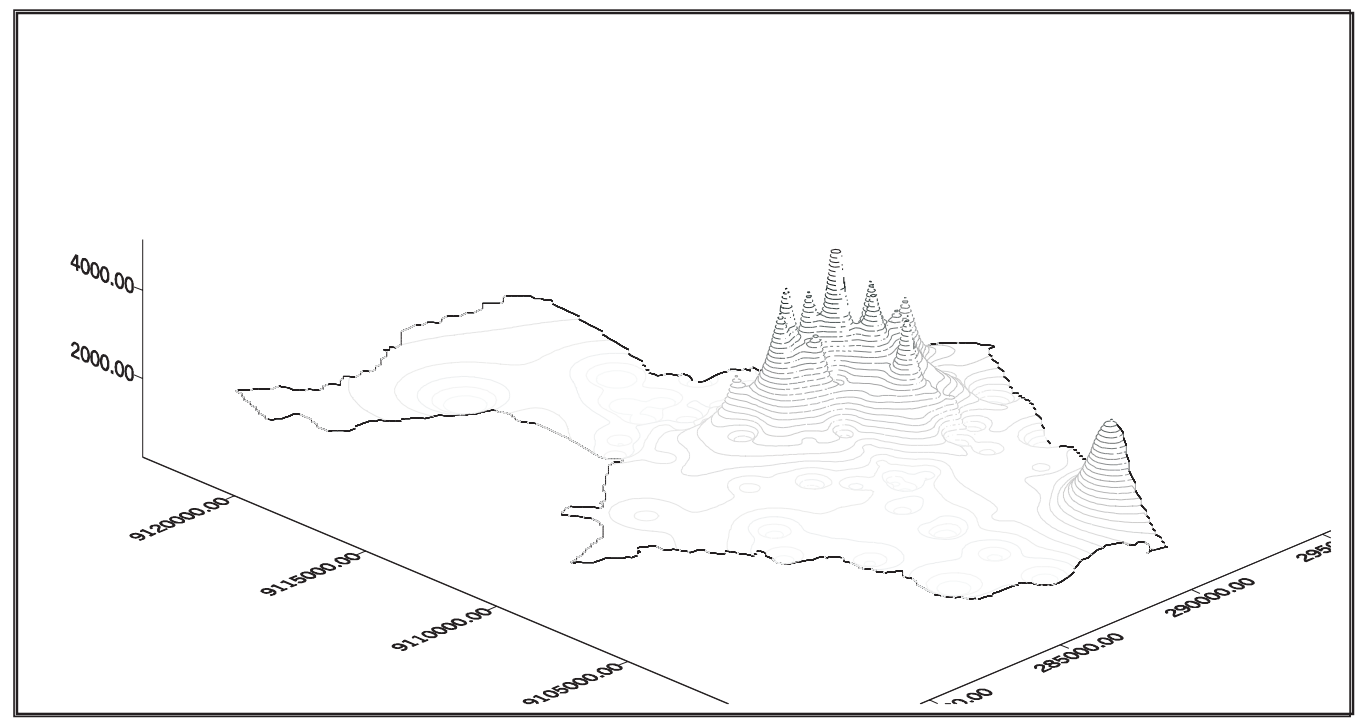

Fonte: IBGE - Censo Demográfico 2000.

Notas: o eixo X representa o sentido oeste-leste, o Y, o sentido sul-norte, e o eixo Z, a renda média do chefe da família em R $\$$. A parte mais elevada da figura representa a região de Boa Viagem na zona sul e a região da Jaqueira na zona centro-oeste.

Para testar a autocorrelação espacial da renda com base na metodologia desenvolvida por Anselin (1988), utilizam-se os testes de dependência espacial, apresentados na Tabela 1, e ajusta-se um polinômio de tendência do segundo grau, obtendo-se os resultados constantes da Tabela 2. 
Tabela 1 - Diagnóstico da dependência espacial para a renda

\begin{tabular}{lcc}
\hline Testes & Valor & Probabilidade \\
\hline MORAN I & 14,1481 & 0,0000 \\
LM ROBUSTO (erro) & 0,5836 & 0,4449 \\
LM ROBUSTO (defasagem) & 3,3699 & 0,0663 \\
\hline
\end{tabular}

Nota: as hipóteses de normalidade e homocedasticidade dos resíduos foram aceitas com uma probabilidade de erro inferior a $1 \%$, quando realizados os testes de Jarque-Bera e Breusch-Pagan, respectivamente.

O teste de Moran I indica a forte presença de efeitos espaciais, uma vez que a hipótese da não existência de dependência espacial foi rejeitada a valores de significância toleráveis. Pelos testes de LM Robusto (erro) e LM Robusto (defasagem), observa-se que apenas o efeito de defasagem espacial é significativo ao nível de 6,63\%. Assim, ajustou-se um modelo de defasagem espacial, ou seja, um polinômio de tendência do segundo grau, considerando-se como variável dependente o logaritmo natural da renda do chefe da família no bairro (LRE), cujos resultados encontram-se na Tabela 2. Os efeitos de interação espacial são confirmados, uma vez que o coeficiente do termo de defasagem espacial da renda, $W_{-} L R E$, é positivo e altamente significativo, com um coeficiente de autocorrelação espacial próximo de $90 \%$, o que indica a presença de dependência espacial fortemente positiva entre a renda média dos chefes de família de um dado bairro e a renda média dos chefes de família dos bairros vizinhos. Assim, pode-se concluir que a renda do bairro não é explicada apenas pela posição geográfica que ele ocupa, mas também pela influência da renda dos bairros vizinhos. Ou seja, o padrão de renda do bairro é um forte determinante na escolha da localização da habitação. Isto é, as pessoas de renda elevada procuram morar em bairros de padrão de renda semelhantes. Este é um fator importante que pode ajudar na elaboração de políticas urbanas, pois o poder público, criando amenidades urbanas, pode desconcentrar a densidade urbana das cidades.

Tabela 2 - Modelo de defasagem espacial para a renda

\begin{tabular}{|c|c|c|c|c|}
\hline Variáveis independentes & Coeficiente & Desvio Padrão & Valor z & Probabilidade \\
\hline W_LRE & 0,898147 & 0,070810 & 12,683809 & 0,000000 \\
\hline CONSTANTE & 0,821191 & 0,466137 & 1,761695 & 0,078121 \\
\hline$x$ & 0,006044 & 0,0289031 & 0,209112 & 0,834361 \\
\hline Y & $-0,041361$ & 0,020357 & $-2,031735$ & 0,042181 \\
\hline$x^{2}$ & $-0,003993$ & 0,0074184 & $-0,538321$ & 0,590355 \\
\hline$Y^{2}$ & $-0,010328$ & 0,0038749 & $-2,665404$ & 0,007690 \\
\hline$X Y$ & $-0,015470$ & 0,0062724 & $-2,466437$ & 0,013646 \\
\hline
\end{tabular}

Pela análise dos coeficientes do polinômio de tendência ajustado na Tabela 2, observa-se que apenas as coordenadas $\mathrm{Y}, \mathrm{Y}^{2}$ e XY, são significantes ao nível de $5 \%$, indicando que as variações de renda, a grande escala espacial, são mais significativas no sentido da coordenada Y, ou seja, na direção norte-sul. Na realidade, é nos bairros da periferia da zona norte onde existe a maior concentração da população de baixa renda, e na zona sul existe o bairro mais populoso, Boa Viagem, com uma das rendas mais elevadas da cidade. 


\section{Dados E ESTIMAÇÕES DO MODELO DE PREÇOS HEDÔNICOS}

Nesta seção, faz-se a estimação empírica do modelo tradicional de preços hedônicos; em seguida, testam-se os efeitos espaciais e, dependendo destes resultados, o modelo espacial mais indicado será estimado, como demonstrado a seguir. ${ }^{21}$

\subsection{Descrição da amostra}

Utiliza-se uma amostra de 228 apartamentos financiados pela Caixa Econômica Federal, na cidade do Recife, no período de junho de 2000 a junho de 2002. Para explicar a variabilidade observada nos preços dos apartamentos, consideram-se como variáveis independentes as seguintes características estruturais: área privativa, idade, conservação, número de quartos sociais, suítes, vagas de estacionamento, pavimentos do edifício, elevadores e unidades da edificação. Como variáveis locacionais, num primeiro momento, foram utilizadas as distâncias de cada edifício, em que estão situados os apartamentos demandados, a dois pólos de influência da cidade, identificados na análise realizada na seção anterior: na zona sul, a praia de Boa Viagem, uma das mais belas praias urbanas do País e, na zona norte, o Parque da Jaqueira, uma grande área verde com pista de cooper, ciclovias e outros entretenimentos, que atraem centenas de pessoas diariamente. Utilizou-se, ainda, a influência do centro histórico da cidade, como tem sido tradicionalmente considerado na literatura. A influência destes pólos foi dada pela distância em quilômetros, obtidas pela montagem de uma matriz de distâncias, construída a partir das coordenadas geográficas de cada edifício, ${ }^{22}$ compatibilizadas com uma planta digitalizada da cidade do Recife, utilizando-se o programa ArcView. Tendo em vista que a renda do bairro é um forte determinante na escolha da habitação, considerou-se a renda média do chefe da família fornecida pelo censo demográfico do IBGE (2000), como uma variável econômica para identificar o padrão socioeconômico do bairro onde está localizado o imóvel. Uma sumarização da amostra encontra-se na Tabela $3{ }^{23}$

Os resultados apresentados na Tabela 3 indicam a existência de uma grande heterogeneidade dos dados, tanto do ponto de vista econômico quanto estrutural e espacial. Os valores de compra dos apartamentos variam de $R \$ 15.000,00$ a $R \$ 298.259,00$, isto é, o mais caro tem valor 20 vezes superior ao mais barato; a área privativa varia de $28,81 \mathrm{~m}^{2}$ a $287,34 \mathrm{~m}^{2}$, isto é, o maior apartamento é 10 vezes superior ao menor em área; há apartamentos de 1 a 4 quartos sociais, desde a condição de ausência de suítes e vagas na garagem, até 3 suítes e 3 vagas, situados em edifícios de 2 a 32 pavimentos, com número de unidades que varia de 4 a 150. Existem na amostra desde edifícios recentemente construídos até aqueles com idades que chegam a 40 anos e em três estágios de conservação: ruim, regular e bom. Em relação à heterogeneidade espacial, o padrão socioeconômico do bairro varia 1,75 a 25,98 salários mínimos. Existem edifícios localizados tanto à beira-mar quanto até 11 km de distância da praia, além da variação de distância em relação aos outros pólos considerados.

21 As regressões foram realizadas no SpaceStat.

22 Medidas em UTM, sigla de Universal Transversa de Mercator.

23 Para o tratamento dos dados será utilizado o sistema SpaceStat, desenvolvido por Anselin (1990). 
Tabela 3 - Descrição da amostra dos dados de apartamentos demandados no Recife

\begin{tabular}{lcrrrrr}
\hline Variáveis & Sigla & Mínimo & \multicolumn{1}{c}{ Média } & \multicolumn{1}{c}{ Mediana } & \multicolumn{1}{c}{ Máximo } & Amplitude \\
\hline Valor da Compra (R\$) & VT & 15000,00 & 53946,90 & 37000,00 & 298259,00 & 283259,00 \\
Renda do Bairro & BA & 1,75 & 9,15 & 6,62 & 25,98 & 24,23 \\
Área Privativa & AP & 28,81 & 83,18 & 71,28 & 287,34 & 258,53 \\
Quartos Sociais & QS & 1,00 & 2,45 & 2,00 & 4,00 & 3,00 \\
Suítes & SU & 0,00 & 0,47 & 0,00 & 3,00 & 3,00 \\
Vagas & VA & 0,00 & 1,08 & 1,00 & 3,00 & 3,00 \\
Pavimentos & NP & 2,00 & 8,27 & 4,00 & 32,00 & 30,00 \\
Elevadores & EL & 0,00 & 0,82 & 0,01 & 4,00 & 4,00 \\
Unidades & UH & 4,00 & 24,38 & 16,00 & 150,00 & 150,00 \\
Idade & ID & 0,00 & 12,20 & 12,00 & 40,00 & 40,00 \\
Conservação & CO & 1,00 & 2,18 & 3,00 & 3,00 & 2,00 \\
Período & PE & 0,00 & 0,69 & 1,00 & 1,00 & 1,00 \\
Distância Praia & DP & 0,01 & 5,35 & 6,29 & 11,43 & 11,42 \\
Distância Centro & DC & 0,46 & 5,68 & 5,87 & 10,12 & 9,66 \\
Distância Jaqueira & DJ & 0,20 & 4,69 & 3,71 & 11,82 & 11,62 \\
\hline
\end{tabular}

Fonte: Tabulação própria a partir dos dados do SISUFOR-CAIXA.

Notas: * Medida em salários mínimos. A variável qualitativa conservação assumiu valor 1 para o estado ruim, 2 para o estado regular e 3 para o estado bom, enquanto que, para a variável período, adotou-se uma dummy com valor zero para os primeiros 12 meses, e 1 para os demais meses em que foram realizadas as contratações.

\subsection{Modelo tradicional de preços hedônicos}

Uma vez observados os dados, realiza-se a estimação empírica da equação (15), utilizando-se o modelo de regressão clássico, tomando-se por base as estimações via MQO, sendo os resultados apresentados na Tabela 4 , a seguir. ${ }^{24}$

Pode-se observar que os sinais obtidos para os coeficientes das variáveis estão coerentes com o mercado, uma vez que há expectativas de que o preço dos apartamentos cresça com o aumento do padrão socioeconômico do bairro em que está localizado, com a quantidade de metros quadrados de área privativa, com o número de quartos sociais, de suítes e de vagas na garagem. Também se espera que as unidades situadas em edificações com maior número de pavimentos, de elevadores e de melhor conservação, sejam mais valorizadas. Contudo, os apartamentos em edifícios mais velhos e com grande número de unidades são mais baratos. Em relação aos pólos de influência, verificase que os apartamentos são mais valorizados na medida em que se aproximam da praia e também do parque da Jaqueira, mas se desvalorizam ao se aproximarem do centro da cidade, comprovando mais uma vez a estrutura multicêntrica da cidade do Recife. Os coeficientes da grande maioria das variáveis se mostraram estatisticamente significantes ao nível de $1 \%$, com exceção das variáveis Suítes, Vagas na Garagem e Período. O modelo apresenta um alto poder explicativo com coeficiente de determinação de 0,90 , e a hipótese nula de que o conjunto de variáveis explicativas adotadas

24 Com o objetivo de testar a estabilidade estrutural dos parâmetros nas zonas norte sul da cidade, o teste de Chow-Wald, ampliado por Anselin (1990), foi utilizado. Os resultados obtidos indicam que a hipótese nula de igualdade de coeficientes em ambas as regiões da cidade não pode ser rejeitada a qualquer nível razoável de significância. Dessa forma, pode-se considerar os preços implícitos das características da habitação como constantes ao longo de toda a região abrangida pela pesquisa. 
não é importante para explicar a variabilidade observada nos preços dos apartamentos é fortemente rejeitada, quando utilizado o teste $\mathrm{F}$.

Tabela 4 - Modelo tradicional de preços hedônicos na forma funcional semilog

\begin{tabular}{lrccc}
\hline Variáveis independentes & Coeficiente & Desvio Padrão & Estatística t & Probabilidade \\
\hline Constante & 10,152 & 0,127 & 79,658 & 0,000 \\
Renda do Bairro & 0,012 & 0,004 & 3,244 & 0,001 \\
Área Privativa & 0,006 & 0,001 & 9,050 & 0,000 \\
Quartos Sociais & 0,072 & 0,029 & 2,452 & 0,015 \\
Suítes & 0,036 & 0,021 & 1,686 & 0,093 \\
Vagas & 0,047 & 0,034 & 1,397 & 0,164 \\
Pavimentos & 0,021 & 0,004 & 4,833 & 0,000 \\
Elevadores & 0,100 & 0,027 & 3,640 & 0,000 \\
Unidades & $-0,003$ & 0,001 & $-2,957$ & 0,003 \\
Idade & $-0,010$ & 0,002 & $-4,769$ & 0,000 \\
Conservação & 0,071 & 0,019 & 3,644 & 0,000 \\
Período & 0,048 & 0,029 & 1,687 & 0,093 \\
Distância Praia & $-0,066$ & 0,017 & $-3,819$ & 0,000 \\
Distância Centro & 0,082 & 0,023 & 3,627 & 0,000 \\
Distância Jaqueira & $-0,077$ & 0,020 & $-3,884$ & 0,000 \\
$R^{2}$ & & & & \\
$R^{2}$ ajustado & 0,9049 & & & \\
AIC & 0,8987 & & & \\
SC & $-97,6167$ & & & \\
Teste F (Probabilidade) & $-46,1766$ & &
\end{tabular}

Notas: AIC significa critério de informação de Akaike e CS é o critério de Scharwz.

Pelos resultados acima apresentados, poderíamos concluir que, mantendo-se as demais condições constantes, os consumidores estão dispostos a pagar a 1,20\% a mais no preço do apartamento para morar em um bairro de padrão socioeconômico com um salário mínimo maior; 0,59\% por cada metro quadrado de área privativa; $7,48 \%$ por um quarto social; 3,63\% por uma suíte; 4,85\% por cada vaga na garagem. Também os apartamentos situados em edificações com maior número de pavimentos são mais valorizados na razão de $2,12 \%$ por pavimento; cada elevador adicional no edifício eleva os preços médios em 10,51\% e para cada nível de melhor conservação há uma valorização de 7,36\%. Por outro lado, os apartamentos se desvalorizam com o acréscimo do número de unidades, na razão de 0,31\%, e com a idade a uma taxa de 0,96\% ao ano. Em relação aos pólos de influência, poderíamos concluir que os apartamentos se desvalorizam a uma taxa de 6,35\% e 7,44\% a cada quilômetro que se distanciam da praia e do parque da Jaqueira, respectivamente, enquanto sofrem um acréscimo no valor, a uma taxa de $8,52 \%$, na medida em que se afastam um quilômetro do centro da cidade.

Apesar dos bons resultados obtidos na estimação, deve-se notar que uma importante questão ainda não foi levada em consideração: a dimensão espacial. A rigor, nada se pode concluir a respeito dos parâmetros deste modelo antes de testar a autocorrelação espacial, pois, caso ela exista, como 
mencionado acima, os parâmetros estimados podem apresentar problemas de tendenciosidade, inconsistência ou ineficiência.

\subsection{Diagnóstico da dependência espacial}

Para detectar a presença de efeitos espaciais nos dados, são utilizados os testes LM Robusto (erro) e LM Robusto (defasagem), realizados sobre os resíduos de mínimos quadrados do modelo estimado na Tabela 4. Para a realização destes testes, trabalhou-se com uma matriz de vizinhança, W, como definida por Anselin (1988), onde cada elemento da matriz, $\mathbf{w}_{\mathrm{ij}}$, representa o inverso da distância entre os edifícios observados, medida em hectômetros. Neste caso, considerou-se peso 1 para edifícios distantes até $1 \mathrm{hm}$ e zero para edifícios com distâncias superiores a $3 \mathrm{hm}$, ou seja, considerou-se que a influência dos efeitos microlocalizativos é desprezível a partir de distâncias superiores a $3 \mathrm{~km}$, com base nas análises dos variogramas. ${ }^{25}$ Em seguida, a matriz W é padronizada por linha.

A Tabela 5 apresenta os resultados para os testes de dependência espacial para a amostra. O teste LM Robusto (defasagem) indica um forte efeito de defasagem espacial nos preços dos apartamentos, uma vez que o teste é significante a menos de $2 \%$. Ou seja, o teste indica a existência de um efeito vizinhança na determinação dos preços dos apartamentos nessa cidade.

\section{Tabela 5 - Diagnóstico da dependência espacial}

\begin{tabular}{lccc}
\hline Teste & MI/DF & Valor & Probabilidade \\
\hline LM Robusto (erro) & 1 & 0,469 & 0,493 \\
LM Robusto (defasagem) & 1 & 5,508 & 0,019 \\
\hline
\end{tabular}

Diante dessa possibilidade de autocorrelação espacial, ou efeito vizinhança, a seguir, estima-se o modelo de defasagem espacial de preços hedônicos, equação 20. Os resultados da estimação estão explicitados na Tabela 6.

Os resultados da estimação espacial da Tabela 6 indicam que o efeito espacial, $\rho$, é positivo e estatisticamente significante a menos de $1 \%$, implicando a existência de um forte efeito de defasagem espacial positiva na amostra. Isto é, neste caso, a formação dos preços de mercado não é explicada apenas pelas suas características estruturais e locacionais tradicionalmente consideradas, mas também depende dos preços dos apartamentos vizinhos, isto é, são influenciados positivamente pelos vizinhos. Pode-se observar que houve modificações nos resultados quando comparados com os apresentados na Tabela 4, fato não surpreendente, uma vez que o efeito espacial é significante. Verifica-se que os coeficientes das variáveis tiveram seus desvios padrões reduzidos, notadamente nas variáveis Vagas e Período, que sofreram alterações de 16,37\% para 10,02\% e 9,30\% para 5,27\%, respectivamente. Observe, também, que as alterações mais significativas nas magnitudes dos coeficientes ocorreram no intercepto e nas variáveis relacionadas à localização, que tiveram seus valores reduzidos. Outro aspecto importante é que os valores obtidos de AIC e SC são bem inferiores aos encontrados no modelo tradicional, o que mostra a melhoria no modelo ajustado pela nova metodologia.

25 Ver variograma apresentado na Figura 1. 
Tabela 6 - Resultados do modelo de defasagem espacial

\begin{tabular}{lcccc}
\hline Variáveis independentes & Coeficientes & Desvio padrão & Valor z & Probabilidade \\
\hline W_LVT & 0,2061 & 0,0741 & 2,7796 & 0,0054 \\
Constante & 7,8926 & 0,8267 & 9,5473 & 0,0000 \\
Renda do Bairro & 0,0072 & 0,0039 & 1,8445 & 0,0651 \\
Área Privativa & 0,0059 & 0,0006 & 9,4066 & 0,0000 \\
Quartos Sociais & 0,0648 & 0,0280 & 2,3150 & 0,0206 \\
Suítes & 0,0333 & 0,0201 & 1,6517 & 0,0986 \\
Vagas & 0,0531 & 0,0323 & 1,6440 & 0,1002 \\
Pavimentos & 0,0215 & 0,0041 & 5,2095 & 0,0000 \\
Elevadores & 0,0925 & 0,0262 & 3,5372 & 0,0004 \\
Unidades & $-0,0030$ & 0,0010 & $-3,0031$ & 0,0027 \\
Idade & $-0,0097$ & 0,0019 & $-5,0422$ & 0,0000 \\
Conservação & $-0,0687$ & 0,0185 & $-3,7140$ & 0,0002 \\
Período & 0,0528 & 0,0273 & 1,9373 & 0,0527 \\
Distância Praia & $-0,0415$ & 0,0187 & $-2,2233$ & 0,0262 \\
Distância Centro & 0,0615 & 0,0227 & 2,7034 & 0,0069 \\
Distância Jaqueira & $-0,0587$ & 0,0202 & $-2,8998$ & 0,0037 \\
R & & & & \\
AIC & 0,91 & & & \\
SC & $-102,70$ & & & \\
\hline
\end{tabular}

Notas: AIC significa critério de informação de Akaike e CS é o critério de Scharwz.

Pelos resultados obtidos, verifica-se que a consideração da questão espacial, com base em distâncias a pólos de influência, ou dividindo o espaço em regiões ou zonas, não é capaz de explicar completamente as variações dos preços em relação à localização da habitação. Isto porque não são apenas os pólos que afetam os preços dos apartamentos, mas existe uma verdadeira interação entre os dados, de forma que cada edifício funciona com um micropólo de influência sobre os seus vizinhos. A solução para estimação segura da equação de preços hedônicos, na presença de efeitos espaciais, deve ser feita com base na econometria espacial, em que a variável de defasagem espacial, no caso em análise W_LnP, que leva em consideração todas as interações espaciais possíveis entre os dados, serve como proxy para variáveis locacionais não consideradas no modelo.

\section{CONCLUSÕES}

A hipótese comumente imposta na análise dos preços de que as observações são independentes não parece se confirmar na avaliação de imóveis. Os dados nesse tipo de análise são distribuídos no espaço, gerando a possibilidade de que existam erros de medidas em relação à exata localização do imóvel, como também efeitos de interação, difusão e "spillovers" espaciais. Estas razões são causadoras de um fator adicional, conhecido como autocorrelação espacial, que deve ser considerado no modelo de regressão tradicionalmente utilizado na engenharia de avaliações, sob pena de se 
incorrer em problemas de estimativas tendenciosas, ineficientes e/ou inconsistentes. Neste caso, a metodologia tradicionalmente adotada torna-se inadequada, podendo levar o avaliador à obtenção de resultados não confiáveis, devendo ser substituída pela nova metodologia denominada regressão espacial.

O presente trabalho teve como objetivo analisar a determinação de preços no mercado de imóveis do Recife, utilizando uma amostra de 232 imóveis de diversos bairros da cidade.

Os resultados encontrados estão de acordo com a literatura em termos de sinais dos coeficientes. Entretanto, dado que os testes de correlação espacial indicaram a presença de dependência espacial, os resultados iniciais, sem considerar tal dependência, eram viesados. Assim sendo, o modelo de autocorrelação espacial foi estimado e seus resultados confirmaram a importância dos efeitos espaciais na amostra.

Pelos resultados obtidos, verifica-se que a consideração da questão espacial, com base em distâncias a pólos de influência, ou dividindo o espaço em regiões ou zonas, não é capaz de explicar completamente as variações dos preços em relação à localização da habitação. Isto porque não são apenas os pólos que afetam os preços dos apartamentos, mas existe uma verdadeira interação entre os dados, de forma que cada edifício funciona com um micropólo de influência sobre os seus vizinhos. A solução para estimação segura da equação de preços hedônicos, na presença de efeitos espaciais, deve ser feita com base na econometria espacial, em que a variável de defasagem espacial, no caso em análise $\mathbf{W} \_\mathbf{L n P}$, que leva em consideração todas as interações espaciais possíveis entre os dados, serve como proxy para variáveis locacionais não consideradas no modelo.

Tendo em vista a autocorrelação espacial positiva nos dados, pode-se concluir ainda que as negociações de compra dos apartamentos não ocorrem de forma independente, como considerado no modelo de preços hedônicos tradicionalmente estimado, mas que existe uma verdadeira interação entre estes preços, de forma que uma negociação de um apartamento por um preço elevado irá gerar uma influência de elevação nos preço dos imóveis vizinhos.

\section{REFERÊNCIAS BIBLIOGRÁFICAS}

Anselin, Luc. Spatial econometrics: methods and models. Dordrecht: Kluwer Academic, 1988.

. Lagrange Multiplier test diagnostics for spatial dependence and spatial heterogeneity. Geographical Analysis, v. 20, p. 1-17, 1988b.

SpaceStat: a program for the statistical analysis of spatial data. Santa Barbara, CA: Departament of Geography and Departament of Economics, University of California, 1990.

.; Rey S. Properties of tests for spatial dependence in linear regression models. Geographic Analysis, v. 23, p. 112-31, 1991.

.; Bera, A. Spatial dependence in linear regression models with an introduction to spatial econometrics. In: Ullah, A.; Giles, D. (Ed.) Handbook of applied economic statistics. Giles: Marcel Dekker, 1998.

Basu, S.; Thibodeau, T. Analysis of spatial autocorrelation in house prices, Journal of Real Estate Finance and Economics, v. 17, p. 61-85, 1998.

Box; Cox An analysis of transformations. J. R. Statistic. Soc., B. Series, v. 26, p. 211-52, 1964.

Can, A., The measurement of neighborhood dynamics in urban housing prices. Economic Geography, v. 66, p. 254-272, 1990. 
Can, A. Specification and estimation of hedonic housing price models. Regional Science and Urban Economics, v. 22, p. 453-477, 1992.

Case, A. C., Spatial patterns in house demand. Econometrica, v. 59, p. 953-965, 1991.

.; Rosen, H. S.; Hines, J. R. Budget spillovers and fiscal policy interdependence: evidence from the states. Journal of Public Economics, v. 52, p. 285-307, 1993.

Instituto Brasileiro de Geografia e Estatística - IBGE. Censo demográfico. 2000.

Cliff, A. D.; Ord, John K. Spatial autocorrelation. London: Pion, 1973.

.; Ord, John K. Space-time modeling with an application to regional forecasting. Trans. Inst. Brit. Geog., v. 64, p. 119-128, 1975.

.; Ord, John K. Spatial processes: models and applications. London: Pion, 1981.

Court, A. T. Hedonic price indexes with automotive examples. In: The dynamics of automobile demand New York: General Motors, 1939, p. 98-119.

Dantas, R. A.; Cordeiro, G. M. Avaliação de imóveis utilizando metodologia científica. V Congresso Brasileiro de Engenharia de Avaliações e Perícias. Recife, 1986

Uma nova metodologia para avaliação de imóveis utilizando modelos lineares generalizados. Revista Brasileira de Estatística, n. 191, 27-46, 1988.

. Uma avaliação do mercado de apartamentos do Recife utilizando modelos lineares generalizados. $19^{\circ}$ Congresso Panamericano de Avaliações. Margarita, Venezuela, 2000.

. Evaluation of the brazilian city of Recife's condominium market using generalized linear models The Appraisal Jornal, v. LXIX, n. 3, USA, 2001.

Dantas, R. A. et al. Uma nova metodologia para avaliação de imóveis utilizando regressão espacial, Anais do XI Congresso Brasileiro de Engenharia de Avaliações e Perícias. 2001.

Dantas, R. A. et. al. La importancia de la regresión espacial en la tasación de inmuebles. I Congreso Internacional en Tasación y Valoración. Espanha, 2002.

Dantas, R. A.; Rocha, F. S.; Magalhães, A. La importancia de la regresión espacial en la tasación de inmuebles. Anais del I Congreso Internacional en Tasación y Valoración. Valencia, Espanha, 2002a.

. Modelos espacilales aplicados en la tasación de inmuebles. Anais del XX Congreso Panamericano de Valuación. Buenos Aires, Argentina, 2002b.

Dubin, R. Spatial autocorrelation and neighborhood quality. Regional Science and Urban Economics, v. 22, p. 433-452, 1992

Ermisch, J. F.; Findlay, J.; Gibb, K. The price elasticity of housing demand in britain issues of sample selection. Journal of Housing Economics, v. 5, p. 64-86, 1996.

Florax, R.; Rey, S. The impact of misspecified spatial interaction in linear regression models. In: Anselin, L.; Florax, R. (Ed.) New Direction in Spatial Econometrics. 1995.

Garcia, Jose Miguel et al. Una nueva metodología en la valoración de inmuebles mediante modelos espaciales de regresión. Anais del I Congreso Internacional en Tasación y Valoración. Valencia, Espanha, 2002.

Goodman, A. C. Andrew court and the invention of hedonic price analysis. Journal of Urban Economics, v. 44, p. 291-298, 1998.

Guervós, R. C. Aproximación al valor de la vivenda aplicación a la ciudad de Granada. Editorial Universidade de Granada, 1999.

Griliches, Zvi. Hedonic price indices for automobiles: an econometric analysis of quality changes. In: Griliches, Z. (Ed.) Price indexes and quality change. Cambridge, Mass: Harvard University Press, 1971.

Halvorsen, R.; Palmquist, R. The interpretation of dummy variables in semilogarithmic equations. American Economia Review, v. 70, n. 3, p. 474-475, 1980.

Lucena, J. M. P. O mercado habitacional no Brasil. Tese (Doutorado). EPGE/FGV-RJ, 1985. 
Matheron, G. Les variables regionalisées et leur estimation. Masson, Paris, 1965.

Olmo, J. E. Spatial estimation of housing prices and locational rents. Urban Studies, v. 32, p. 1331-1344, 1995.

.; Guervós, R. Valoración espacial del precio de la vivienda y del suelo mediante el método de Krigeage. Anais del I Congreso Internacional en Tasación y Valoración. Valencia, Espanha, 2002.

Rosen, S. Hedonic prices and implicit markets: product differentiation pure competition. Journal of Political Economy, v. 82, p. 34-55, 1974.

Tiware, P.; Parich, K.; Parikh, J. Effective housing demand in Mumbai (Bombay) metropolitan region. Urban Studies, v. 36, n. 10, p. 1783-1809, 1999. 